\title{
Site characterisation at Mount Stromlo Observatory: the first results
}

\author{
Visa Korkiakoski ${ }^{\mathrm{a}, \mathrm{b}}$, Doris Grosse ${ }^{\mathrm{a}, \mathrm{b}}$, Elliott Thorn ${ }^{\mathrm{a}, \mathrm{b}}$, Michael Copeland ${ }^{\mathrm{a}, \mathrm{b}}$, Francis Bennet ${ }^{\mathrm{a}, \mathrm{b}}$, \\ James Osborn ${ }^{\mathrm{c}}$, Josephine Munro ${ }^{\mathrm{a}, \mathrm{b}}$, Piotr Piatrou ${ }^{\mathrm{a}, \mathrm{b}}$, Francois Rigaut ${ }^{\mathrm{a}, \mathrm{b}}$, and Celine \\ d'Orgeville ${ }^{\mathrm{a}, \mathrm{b}}$ \\ ${ }^{a}$ Research School of Astronomy and Astrophysics, Australian National University, Mount \\ Stromlo Observatory, Weston Creek, Australia \\ ${ }^{\mathrm{b}}$ SERC Limited, Mount Stromlo, Weston Creek, Australia \\ ${ }^{\mathrm{c}}$ Department of Physics, Centre for Advanced Instrumentation, University of Durham, South \\ Road, Durham DH1 3LE, UK
}

\begin{abstract}
We present the status of the site-characterisation campaign that has been started to aid the development of new adaptive optics systems at the Mount Stromlo Observatory. Our goal is to use a particularly designed stereoSCIDAR system that uses a roof prism to separate beams from a double-star system to obtain two isolated pupil images on a single detector. Currently, the instrument is partially finished and operates as a generalised SCIDAR. The first results and their analysis are shown. We see that the instrument is working as intended: the data shows no sign of harmful artefacts and the atmospheric profiles follow expected patterns. In addition, we show a comparison of two possible methods to post-process Stereo-SCIDAR data: classical SCIDAR approach and a peak-detection based algorithm. Both algorithms agree on the broad features of the turbulence, but the latter is able to reach finer altitude resolution.
\end{abstract}

Keywords: generalised SCIDAR, stereo-SCIDAR, atmospheric turbulence profile measurement, site characterisation, data post-processing

\section{INTRODUCTION}

The Research School of Astronomy and Astrophysics (RSAA) at the Australian National University (ANU) at Mount Stromlo Observatory (MSO), as a partner of Space Environment Management Cooperative Research Centre (SERC), is currently developing adaptive optics (AO) systems for space situational awareness, specifically space debris tracking and space debris orbit perturbation. ${ }^{1-4}$

As a part of the SERC collaboration, we will design and implement two independent AO systems on the Electro Optic Systems (EOS) $1.8 \mathrm{~m}$ telescope: one for imaging capabilities and another with tracking and pushing option. The systems are designed to be operated with and without a sodium laser guide star.

The knowledge of typical atmospheric conditions at MSO is critical for optimal design of the AO systems installed there. In addition, the knowledge of turbulence will make it possible to understand how well the systems are performing with respect to their theoretical limitations. Therefore, we are implementing a campaign to measure typical atmospheric conditions at the MSO site, using a specifically designed stereo-SCIDAR instrument. ${ }^{3,4}$

The site monitoring effort we are conducting will also be useful outside its immediate goal. We are testing a stereo-SCIDAR design that has potential to reduce the costs and complexity of site monitoring. This could make it an interesting option as an auxiliary instrument for the laser tomography adaptive optics applications with the extremely large telescopes. Furthermore, we conduct research on alternative types to process SCIDAR measurements to extract maximal amount of information about the atmospheric turbulence.

Further author information: (Send correspondence to Visa Korkiakoski)

Visa Korkiakoski: E-mail: visa.korkiakoski@anu.edu.au, Telephone: + 61261256084 
This paper gives an update of the site monitoring project. Section 2 summarises the specifications and design of our SCIDAR instrument. Section 3 describes the experiments we have conducted. Section 4 shows preliminary results with our data post processing methods. Finally, conclusions are shown in Section 5.

\section{SYSTEM DESIGN}

The stereo-SCIDAR was introduced by H.W. Shepherd, ${ }^{5}$ and its theory and principles are discussed in more detailed in numerous earlier publications.

The details of our SCIDAR instrument have been reported before. ${ }^{3,6}$ Here, we give a brief summary.

In short, the general SCIDAR images beams (pupil images at a conjugate altitude) from two closely separated stars, as illustrated left in Figure 1. In a stereo-SCIDAR, however, the beams are separated such that the pupil images do not overlap. This can be achieved by two separate detectors ${ }^{5}$ or by inserting a roof prism into the optical path (as in our design ${ }^{3}$ ), illustrated right in Figure 1 - we use a double roof prism designed to minimise chromatic aberrations. The imaged beams can then be cross-correlated and processed to obtain vertical turbulence profiles; spatiotemporal cross-correlations will yield the wind profiles as well.

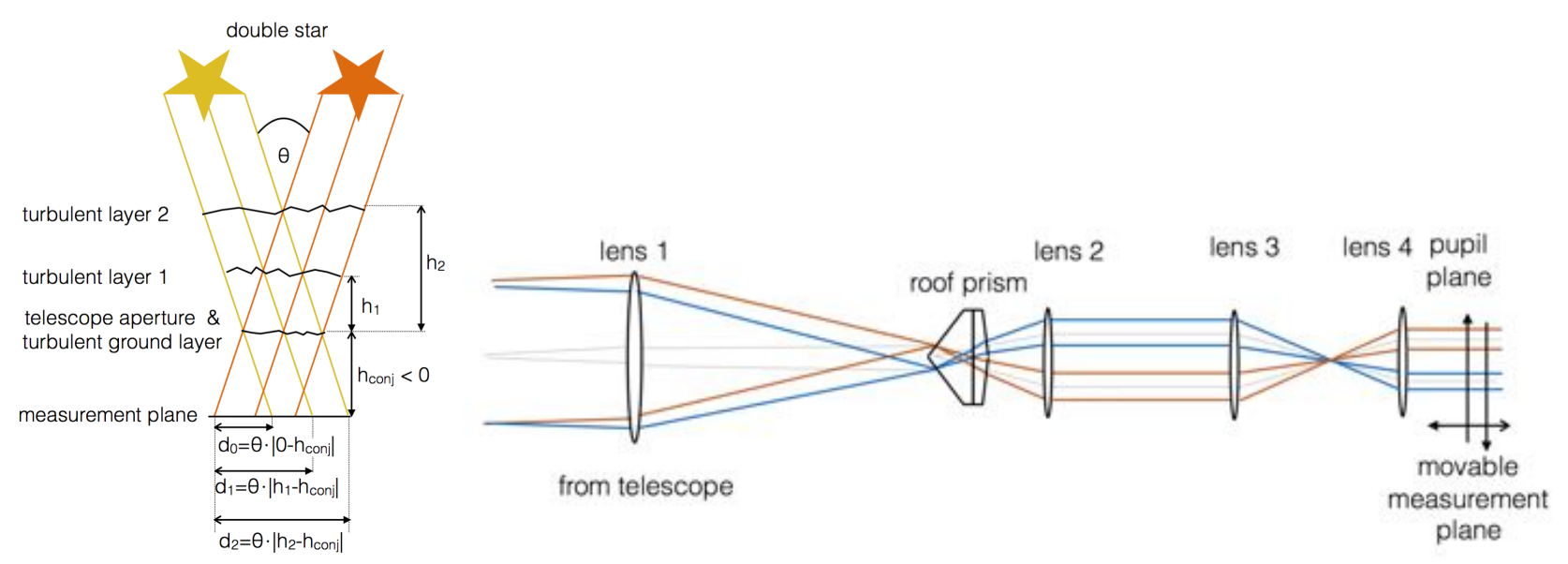

Figure 1: Left: principle of generalised SCIDAR. Phase aberrations from a distinct turbulent layer 1 at height $h_{1}$ through which light shines from a double star with angular separation $\theta$ will be separated by distance $\theta\left|h_{1}-h_{\text {conj }}\right|$. Aberrations from layer 2 will be separated by $\theta\left|h_{2}-h_{\text {conj }}\right|$ and from the ground layer by distance $\theta\left|0-h_{\text {conj }}\right|$. Hence a turbulent layer at height $\mathrm{h}$ will be separated by $\theta\left|h-h_{\text {conj }}\right|$. Right: schematic of optical design of generalised stereo-SCIDAR. The additional roof prism refracts the beams, letting them diverge with the appropriate angle so that the following lens system re-images the scintillation patterns collimated and fully separated. ${ }^{3}$

We have currently implemented a first stage of the setup. The roof prism is not yet installed, so the system works as a generalised SCIDAR. A three-dimensional model of our instrument in shown in Fig. 2.

Our SCIDAR instrument has been installed in the coude lab of EOS 1.8 telescope, sharing the same optical path to telescope as our AO systems. The SCIDAR is designed to operate intermittently - when telescope schedule permits - over the next year.

\section{EXPERIMENTS WITH GENERAL SCIDAR}

This section describes the experiments we have conducted to make sure the intermediate generalised SCIDAR is functioning as expected.

The tests were done on 13th June, 2017, after about 19:30, shortly after sunset at the MSO site. We observed two different double star systems, with parameters as shown in Table 1. These preliminary results have been reported before in ${ }^{6}$ here we give a bit more detailed description. 


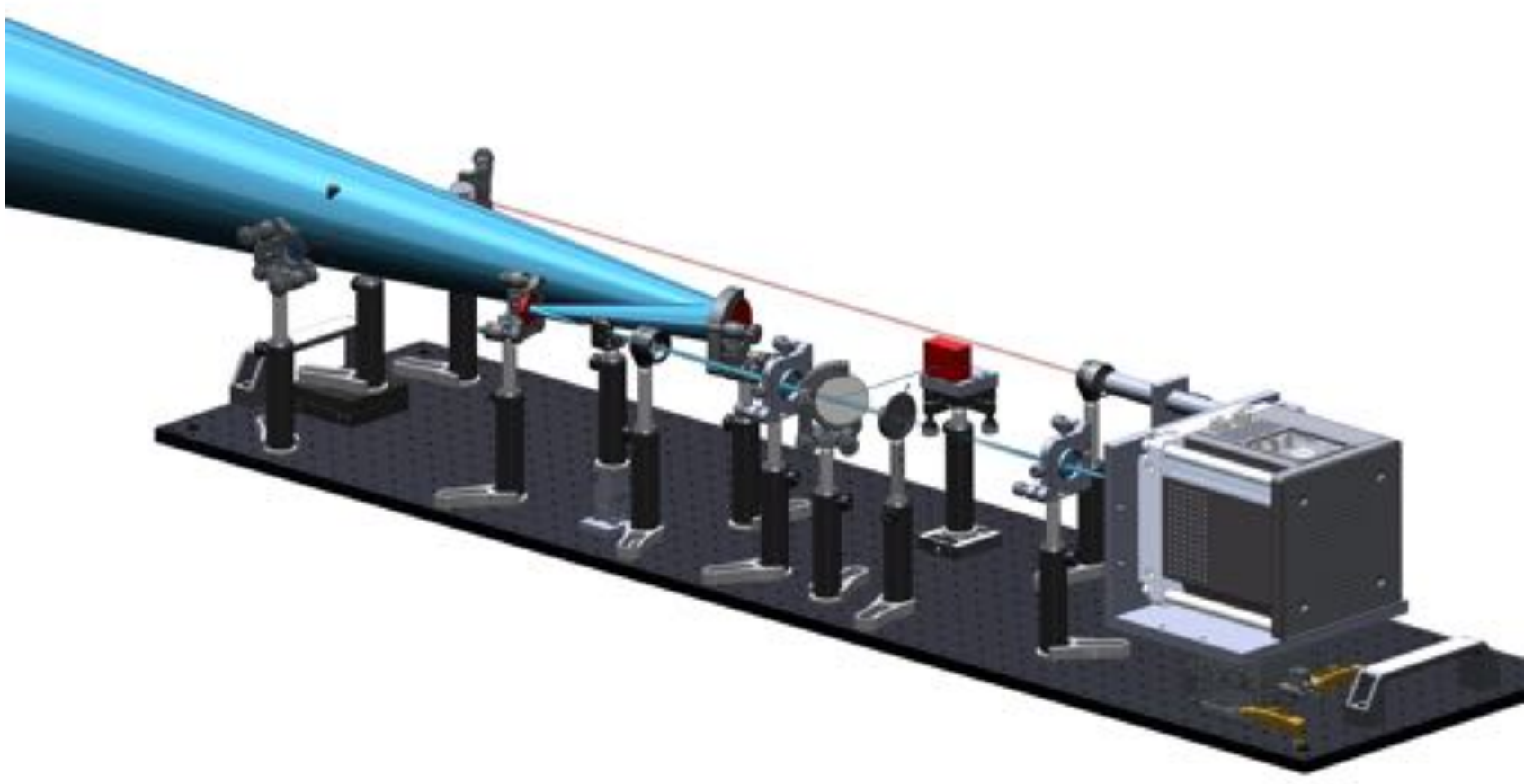

Figure 2: A 3D model of the Generalized SCIDAR instrument.

Table 1: Double stars used with experiments

\begin{tabular}{|llllll|}
\hline Name & Magnitude 1 & Magnitude 2 & Separation & Camera Framerate & conjugation \\
\hline \hline DUN 252 & 1.25 & 1.55 & $3.9 "$ & $250 \mathrm{~Hz}$ & ground \\
DUN 252 & 1.25 & 1.55 & $3.9 "$ & $250 \mathrm{~Hz}$ & $-500 \mathrm{~m}$ \\
PZ 4 & 5.1 & 5.6 & $10.2 "$ & $250 \mathrm{~Hz}$ & $-500 \mathrm{~m}$ \\
\hline
\end{tabular}

The raw data revealed that the pupil is not fully imaged on the camera, probably due to clipping in the coude path. This is illustrated left in Fig. 3.

In addition, we saw that the laser located in the laboratory, used normally for space debris tracking, caused significant periodic changes in the background illumination of our images (see the right plot in Fig. 3). Therefore, we decided to process the images assuming that each image had an unknown bias added on top of the pupil image. After the bias was removed, we proceeded to follow the standard SCIDAR method: calculated auto-correlations, averaged them and fitted impulse response functions to deduce the turbulence.

Yet, as shown in the following, in spite of the issues, we see no significant artefacts in the calculated spatiotemporal cross correlations of the recorded images.

Fig. 4 shows simulated and experimental spatiotemporal cross correlations of the measured pupil images (target with widest separation, conjugation to a small negative altitude).

The simulated cross-correlations are done using a typical 4-layer atmospheric profile. The profile was tailored such that the altitude of the highest layer was roughly equal to the altitude of the highest layer we detected using our instrument. 

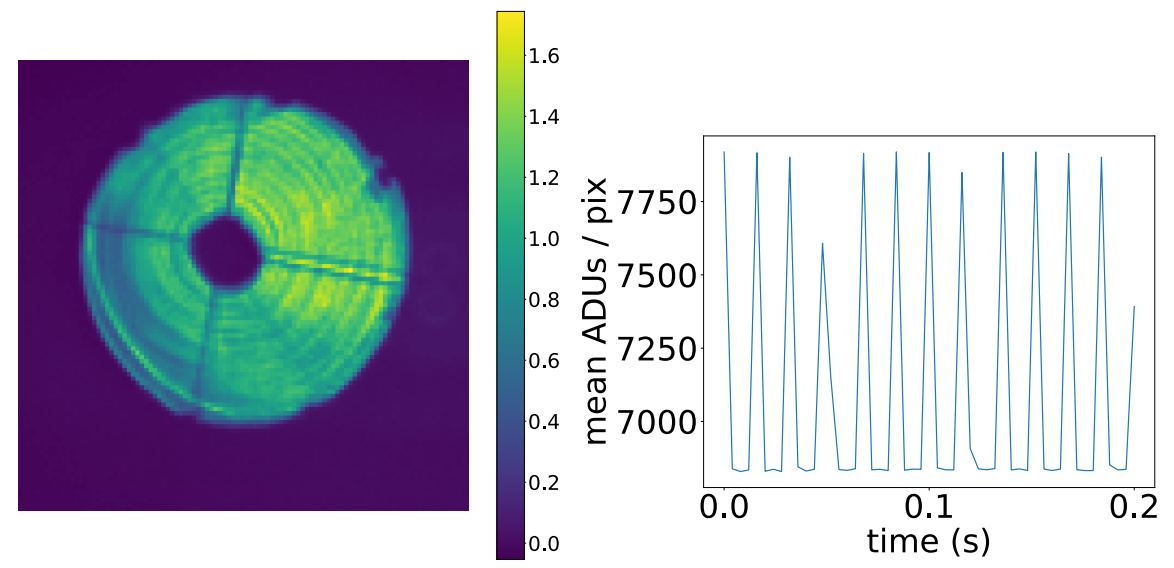

Figure 3: Left: averaged image of the SCIDAR detector (double star DUN 262, ground conjugation). Two overlapping pupil images are seen, with the shadow of the secondary support structure clearly visible. Right: detector counts averaged over the array of $128 \times 128$ pixels are shown as a function of time. Periodic peaks are due to blinking laser and poor shielding of the camera.
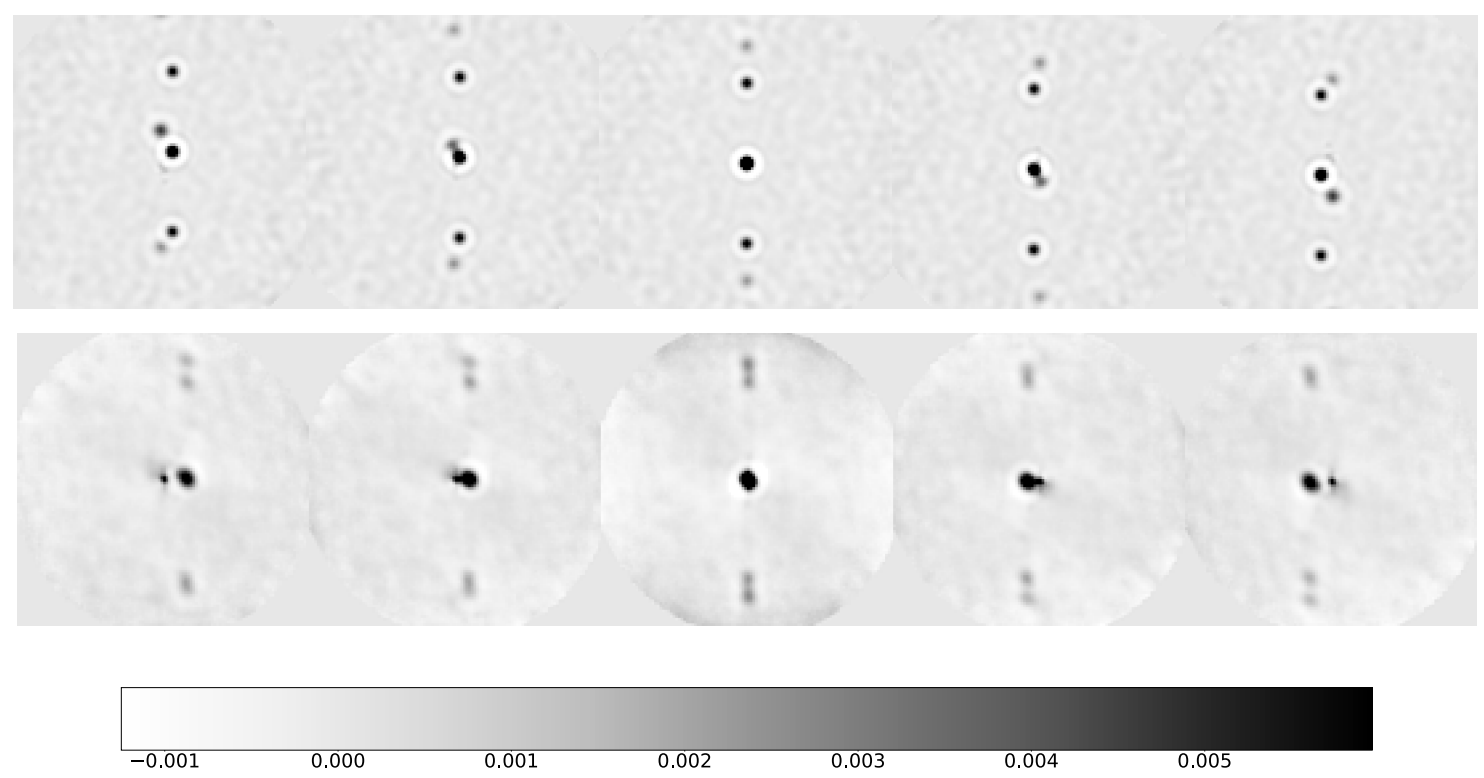

Figure 4: Averaged, spatio-temporal cross-correlations of recorded pupil images. 500 frames used, corresponding to $2 \mathrm{~s}$ of recording. Cross-correlations are rotated such that the double star orientation aligns with positive $\mathrm{y}-$ axis. We show five different time steps, separated by the sampling interval of $4 \mathrm{~ms}$. Up: simulations. Bottom: measurements (target was PZ 4).

Both cross-correlations show clear peaks, which correspond to atmospheric layers. The simulations show that only two upper layers are visible in the averaged auto-correlation, while the impact of ground layers is faintly visible only in the the cross-correlations with \pm 2 frames of delay. This is caused by the fact that the detector was conjugated very close to the ground.

The measured cross-correlations show also two clearly visible high-altitude atmospheric layers. Nothing else is clearly resolved in the auto-correlation, but the cross-correlations reveal a third, compact and relatively strong peak in the middle of the array. This corresponds to strong static low-altitude aberration in the optical path.

The peaks are located differently depending on the time-shift in the cross-correlated arrays, which matches 
the expectation of wind moving a frozen turbulent layer across the aperture. The simulated wind directions do match the actual ones. We simulated different winds for each layer, while in the reality the detected layers travel in the same direction.

The width of the detected auto-correlation peaks is very close to what we would expect from a theoretical Kolmogorov-style turbulence. This is illustrated in Fig 5. It shows that the pupil images we recorded are likely to be free from significant distortions or warps. Thus, we expect no severe artefacts are present due to the coude-path impacts that have clipped the pupil as shown in Fig. 3.
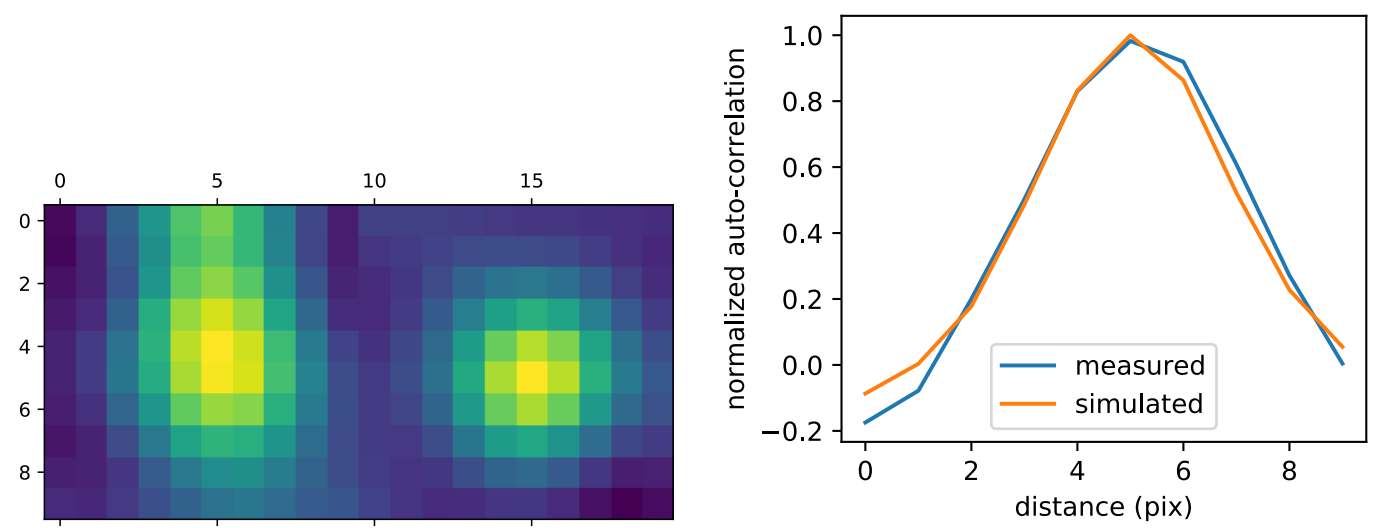

Figure 5: Left: a close-up of the uppermost auto-correlation peaks in Fig. 4 (left is from measurements, right is from simulations). The peaks are normalized to maximum intensity of one. Right: radial cuts of the peaks along x-axis.

Fig. 6 shows the estimated $C_{N}^{2}$ profiles as a function of time and altitude. The profiles have been calculated from auto-correlations using standard SCIDAR post-processing methods. Note that the $\mathrm{x}$-axis is not to scale: time was needed to search for the targets, record bias frames and change conjugate altitude of the detector. The white vertical spaces in between data sets are periods where no data was collected. The three blocks to the left represent the 3.9" separated double star, and the two blocks to the right represent the system with a 10.2" separation.

We see that there is a good match of the layer structure in all the different settings. At least about five distinct layers can be identified.

We estimate the total strength of the turbulence above $1 \mathrm{~km}$ to correspond to a seeing, where the Fried parameter $r_{0}$ at $500 \mathrm{~nm}$ is between $0.3 \mathrm{~m}$ and $0.4 \mathrm{~m}$.

\section{DATA PROCESSING WITH EXPERIMENTAL STEREO-SCIDAR DATA}

Although our SCIDAR instrument is not yet upgraded to a stereo mode, we have developed a unique dataprocessing method for it. ${ }^{4}$ This section illustrates the potential of the Stereo-SCIDAR data processing by applying it on data collected with the ING telescope on La Palma.

We have data for two full nights, provided by J. Osborn. Fig. 7 shows the raw atmospheric profiles during one night, calculated by two methods. The first method uses a conventional approach: fit theoretical 1-D models to cross-sections of auto-correlation arrays and estimate the wind-speeds by separately inspecting of cross-correlation arays. The second method applies a joint method relying on purely peak detection when adjusting the weights of the turbulent layers.

We highlight the following observations:

- Altitude resolution in the joint method is superior. While the conventional SCIDAR approach is limited to resolving peaks in the auto-correlation, a joint method is limited only by the accuracy of fitting lines to 


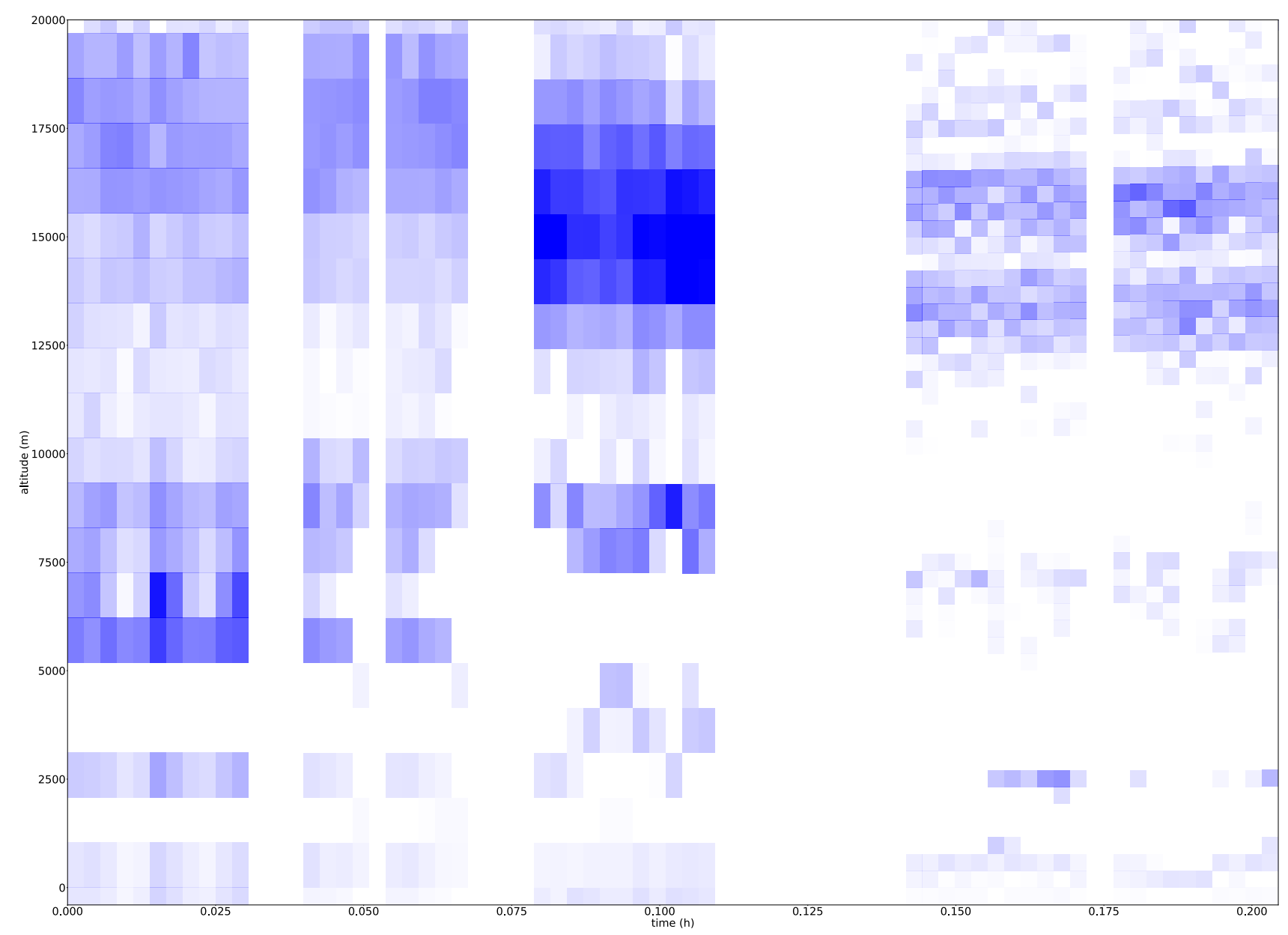

Figure 6: Strength of the turbulence $\left(C_{n}^{2}\right)$ as a function of time and altitude.

peaks that are deemed to represent a moving layer. This is obvious in particular in the "crowded" lower altitudes.

- In general, there is a good match between the two algorithms. There is a consensus of the big features of the atmosphere.

- In some cases, the joint method is able to consistently detect turbulence where the traditional approach does not. For instance, at 13h, below $2000 \mathrm{~m}$. We are still investigating this issue.

We also verified that the total seeing, averaged over time or altitude is in a good agreement between those two processing methods. This is illustrated further by Fig. 8, which shows the strength of the turbulence during both observation nights.

We see that, in general, the both methods have a good agreement on the turbulence above the ground layer. The joint method gives a much higher values on the static ground layer, which could also be caused by issues in the way the cross-correlation arrays are created. We are still investigating the issue.

\section{CONCLUSIONS}

We have reported the status of our site monitoring efforts at Mount Stromlo Observatory. We are aiming to demonstrate that the atmospheric turbulence can be measured with an excellent accuracy using a Stereo-SCIDAR 

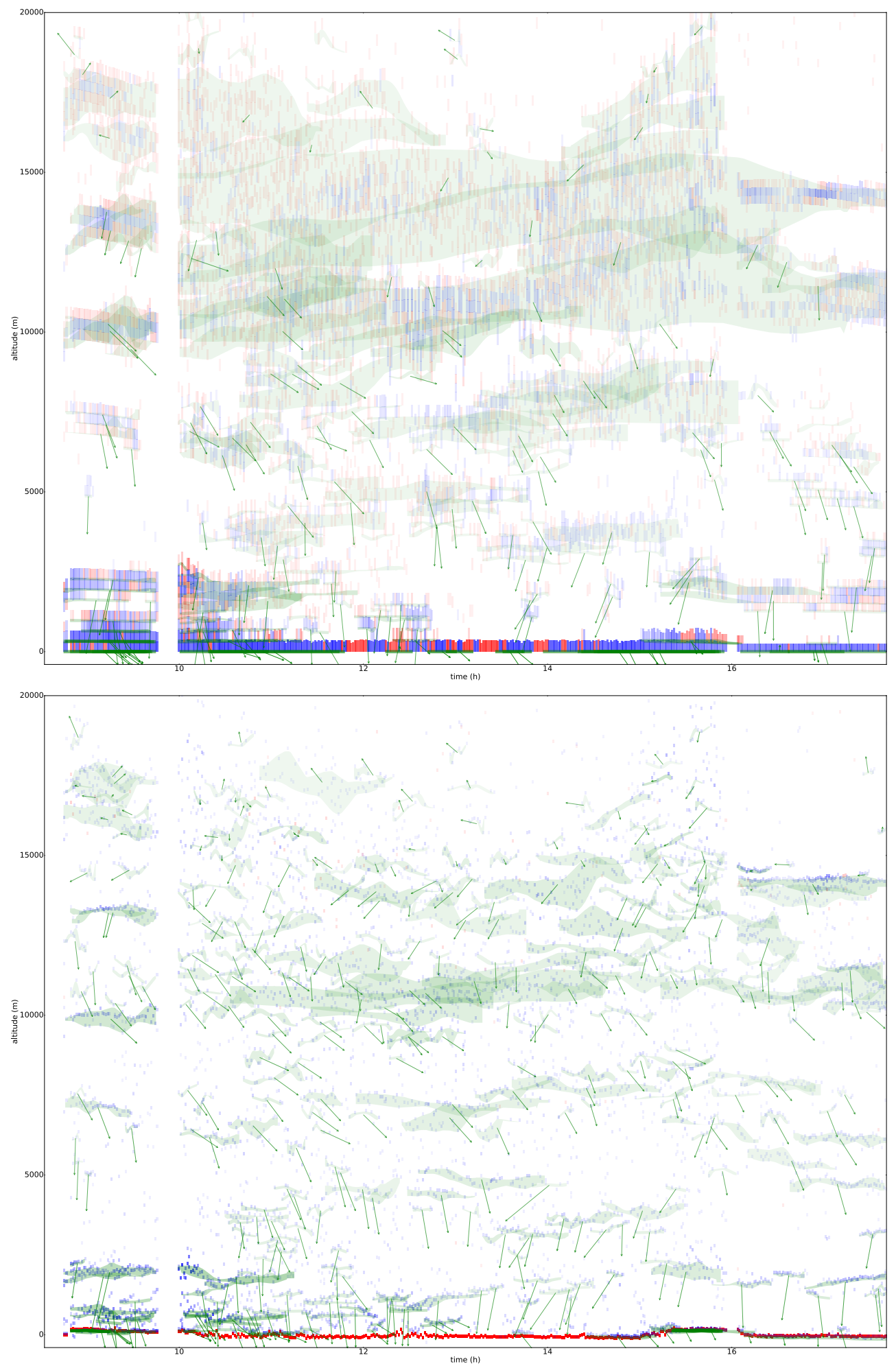

Figure 7: Raw profiles for data recorded in La Palma; turbulence strength as a function of time and altitude. Blue color shows atmospheric turbulence that is moving; shade represents its strength. Red color shows turbulence that is static. Green shade is drawn to encircle the atmospheric layers that we estimate belonging together. Green arrows show the wind speed - the arrow length represents the wind speed. Up: conventional SCIDAR processing used. Bottom: joint method used. 

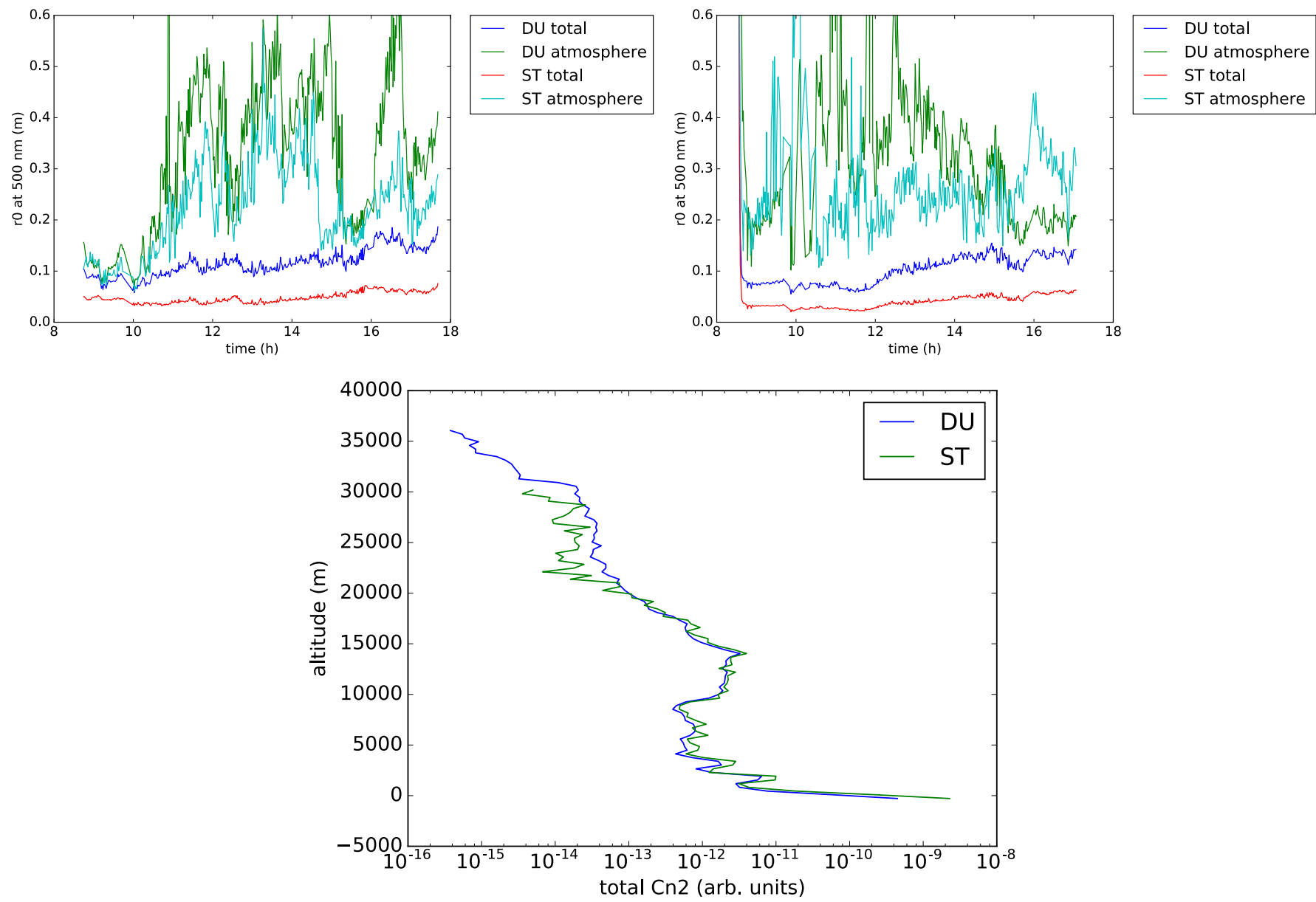

Figure 8: Comparison of two Stereo-SCIDAR post-processing methods. DU represents a conventional method. ST represents the joint method. Up: seeing parameter $r_{0}$ as a function of time; data shown for two nights. Plots referred as "total" are averaged over the whole altitude; plots referred as "atmosphere" are calculated by excluding any static aberrations at the ground level. Bottom: averaged seeing as a function of altitude.

with a single camera. Currently, our instrument is partially finished: we are able to operate it as a generalised SCIDAR. The upgrade to a stereo-mode will happen during the coming months.

We have shown that the instrument currently functions as intended. A careful inspection of the collected data shows no signs of harmful artefacts. The retrieved atmospheric altitude profiles appear plausible.

A proper analysis, however, would require a cross-check with another independent site-profiling instrument, such as SLODAR or DIMM. Such experiments will be conducted at a later stage.

In addition, we have included a comparison between two alternative ways (described in more detail in ${ }^{4}$ ) to post-process Stereo-SCIDAR measurements from ING at La Palma. The comparison indicates that both methods tend to agree on the broad features of the turbulence profile, but an approach based on peak detection has a much higher altitude resolution.

\section{ACKNOWLEDGMENTS}

The authors would like to acknowledge the support of the Cooperative Research Centre for Space Environment Management (SERC Limited) through the Australian Government's Cooperative Research Centre Programme. 
James Osborn is grateful to the Science and Technology Facilities Committee (STFC) for financial support (grant reference ST/J001236/1 and the research leading to these results has received funding from the European Community's Seventh Framework Programme (FP7/2013-2016) under grant agreement number 312430 (OPTICON).

\section{REFERENCES}

[1] Bennet, F., Conan, R., D’Orgeville, C., Murray, M., Paulin, N., Price, I., Rigaut, F., Ritchie, I., Smith, C., and Uhlendorf, K., "Adaptive optics for laser space debris removal," in [SPIE Astronomical Telescopes+ Instrumentation], 844744-844744, International Society for Optics and Photonics (2012).

[2] Bennet, F., D’Orgeville, C., Price, I., Rigaut, F., Ritchie, I., and Smith, C., "Adaptive optics for satellite imaging and space debris ranging," in [Proceedings of the Advanced Maui Optical and Space Surveillance Technologies Conference], Ryan, S., ed., 1, 2, The Maui Economic Development Board (2015).

[3] Grosse, D., Bennet, F., Korkiakoski, V., Rigaut, F., and Thorn, E., "Single detector stereo-scidar for mount stromlo," in [Proc. SPIE], 99093D-99093D (2016).

[4] Korkiakoski, V., Osborn, J., Grosse, D., Thorn, E., Piatrou, P., Bennet, F., and Rigaut, F., "Single detector stereo-scidar for mount stromlo: data analysis," in [Proc. SPIE], 99790L-99790L (2016).

[5] Shepherd, H., Osborn, J., Wilson, R., Butterley, T., Avila, R., Dhillon, V., and Morris, T., "Stereo-scidar: optical turbulence profiling with high sensitivity using a modified scidar instrument," Monthly Notices of the Royal Astronomical Society 437(4), 3568-3577 (2013).

[6] Thorn, E., Korkiakoski, V., Grosse, D., Bennet, F., Rigaut, F., and dOrgeville, C., "Stereo-scidar system for improvement of adaptive optics space debris-tracking activities," in [AMOS conference], (2017). 